\title{
Efficacy and Toxicity Assessment of Different Antibody Based Antiangiogenic Drugs by Computational Docking Method
}

\author{
Sayan Mukherjee, ${ }^{1}$ Gopa Chatterjee, ${ }^{1,2}$ Moumita Ghosh, ${ }^{1}$ \\ Bishwajit Das, ${ }^{1,2}$ and Durjoy Majumder ${ }^{1,2}$ \\ ${ }^{1}$ Department of Physiology, West Bengal State University, Berunanpukuria, Malikapur, Barasat, Kolkata 700 126, India \\ ${ }^{2}$ Society for Systems Biology \& Translational Research, No. 103, Block C, Bangur Avenue, Kolkata 700 055, India \\ Correspondence should be addressed to Durjoy Majumder; durjoy@rocketmail.com
}

Received 30 November 2015; Accepted 21 January 2016

Academic Editor: Huixiao Hong

Copyright ( 2016 Sayan Mukherjee et al. This is an open access article distributed under the Creative Commons Attribution License, which permits unrestricted use, distribution, and reproduction in any medium, provided the original work is properly cited.

\begin{abstract}
Bevacizumab and trastuzumab are two antibody based antiangiogenic drugs that are in clinical practice for the treatment of different cancers. Presently applications of these drugs are based on the empirical choice of clinical experts that follow towards population based clinical trials and, hence, their molecular efficacies in terms of quantitative estimates are not being explored. Moreover, different clinical trials with these drugs showed different toxicity symptoms in patients. Here, using molecular docking study, we made an attempt to reveal the molecular rationale regarding their efficacy and off-target toxicity. Though our study reinforces their antiangiogenic potentiality and, among the two, trastuzumab has much higher efficacy; however, this study also reveals that compared to bevacizumab, trastuzumab has higher toxicity effect, specially on the cardiovascular system. This study also reveals the molecular rationale of ocular dysfunction by antiangiogenic drugs. The molecular rationale of toxicity as revealed in this study may help in the judicious choice as well as therapeutic scheduling of these drugs in different cancers.
\end{abstract}

\section{Introduction}

Solid tumor survives by the process of angiogenesis. Angiogenesis is a physiological process by which microvessels are developed around the tumor mass. Tumor cells secrete a variety of tumor associated growth factors (TAF) like VEGF (vascular endothelial growth factor), TGF (transforming growth factor), EGF (epidermal growth factor), PDGF (platelet-derived growth factor), PAI, and TSP-1 to promote the process of angiogenesis $[1,2]$. The attempt to sequester these factors is known as antiangiogenic (AAG) therapy. It is suggested that AAG therapy can supplement ongoing chemotherapy, for example, with docetaxel, platinum based therapies, paclitaxel, vinorelbine, and gemcitabine especially when they have failed or are not tolerated [3]. For several types of cancer, application of different AAG drugs in combination with the conventional MTD (maximum tolerable dosing strategy) chemotherapy has shown positive results with reduced (chemotherapy related) toxicities [4].
In control of tumor growth, anti-VEGF antibody is being developed. Application of Avastin (bevacizumab), the commercially available anti-VEGF, has a remarkable success in the control of tumor growth in different clinical trials. For AAG therapy another antibody molecule known as trastuzumab has developed. Trastuzumab has the ability to inhibit a variety of other angiogenic molecules, namely, transforming growth factor (TGF), Ang-1, PAI-1, and thrombospondin 1 (TSP1) that might also respond to HER signaling [5].

Bevacizumab was the first antiangiogenic drug that was approved by the U.S. Food and Drug Administration (FDA) in 2004 for different metastatic cancers either alone or in combination with standard chemotherapy (Table 1). It is a recombinant humanized monoclonal IgG1 antibody that binds to and inhibits the biologic activity of human vascular endothelial growth factor (VEGF). Bevacizumab contains human framework regions and the complementaritydetermining regions of a murine antibody that binds to VEGF. Bevacizumab is produced in a Chinese Hamster Ovary 
TABLE 1: FDA-approved different antibody based antiangiogenic drugs.

\begin{tabular}{|c|c|c|c|}
\hline Drug (MW) & Drug target & $\begin{array}{l}\text { Recommended dose } \\
\text { (half-life) }\end{array}$ & Types of cancer that are recommended for treatment \\
\hline $\begin{array}{l}\text { Bevacizumab, } \\
\text { available as Avastin } \\
(149 \mathrm{KD})\end{array}$ & VEGF receptor & $\begin{array}{c}\text { IV infusion of } \\
5-10 \mathrm{mg} / \mathrm{kg} \text { body wt. } \\
\text { in every } 2 \text { or } 3 \text { weeks } \\
(\sim 20 \text { days })\end{array}$ & $\begin{array}{l}\text { Metastatic colon, colorectal, cervical and peritoneal cancer with std. } \\
\text { CT; platinum rst. ovarian or fallopian tube cancer; metastatic HER2 } \\
\text { negative breast cancer; renal carcinoma; first-line treatment of } \\
\text { non-small-cell lung cancer; second-line treatment of glioblastoma, } \\
\text { different types of hematological malignancies }\end{array}$ \\
\hline $\begin{array}{l}\text { Trastuzumab, } \\
\text { available as } \\
\text { Herceptin } \\
(145.5 \mathrm{KD})\end{array}$ & HER-2 & $\begin{array}{c}\text { IV infusion of } \\
2-8 \mathrm{mg} / \mathrm{kg} \text { body wt. } \\
\text { in every week ( } 28.5 \\
\text { days })\end{array}$ & $\begin{array}{l}\text { HER-2 overexpressing including ER/PR negative and node positive or } \\
\text { negative breast cancer in combination with either anthracycline based } \\
\text { (paclitaxel or docetaxel) or cisplatin and capecitabine or } \\
\text { 5-fluorouracil or carboplatin chemotherapy; HER-2 positive } \\
\text { metastatic gastric or gastroesophageal junction endocarcinoma } \\
\text { without prior chemotherapy }\end{array}$ \\
\hline
\end{tabular}

IV: intravenous administration; std.: standard; CT: chemotherapy; rst.: resistant; TK: tyrosine kinase; wt.: weight.

mammalian cell expression system. Interaction of bevacizumab with VEGF prevents the interaction of VEGF to its receptors (Flt-1 fms-like tyrosine kinase-1-VEGFR1 receptor and KDR kinase insert domain containing receptorVEGFR2 receptor) on the surface of endothelial cells. This prevents blood vessel proliferation and in response retardation of metastatic tumor growth occurs [6]. However, application of bevacizumab in patients produces several side effects which include arterial thromboembolic events, hypertension, proteinuria, reversible leukoencephalopathy syndrome, skin rash, pulmonary hypertension, mesenteric venous occlusion, gastrointestinal ulcer and perforation, intestinal necrosis, gallbladder perforation, anastomotic ulceration, pancytopenia, necrotizing fasciitis, wound healing complications, osteonecrosis of the jaw, renal thrombotic microangiopathy (manifested as severe proteinuria), PRES (posterior reversible encephalopathy syndrome), hemorrhage, body as a whole polyserositis, and different forms of eye disorders such as increase in intraocular pressure with inflammation; retinal detachment; conjunctival, vitreous, or retinal hemorrhage; vitreous floaters; ocular hyperemia, pain, or discomfort. However, histopathological kidney lesion like glomerular lesion and interstitial nephritis occur less frequently. Anti-VEGF mediated hypertension is not well understood; nitric oxide pathway inhibition, rarefaction, and oxidative stress may be responsible for such pathogenesis $[5,7-10]$.

Trastuzumab is a IgG1 kappa monoclonal humanized antibody, produced in $\mathrm{CHO}$ cell line, approved by FDA in 2006 for treatment regimen containing doxorubicin, cyclophosphamide, and paclitaxel for the adjuvant treatment of women with node-positive, Her-2 overexpressing breast cancer. It is active against the human epidermal growth factor receptor 2 or $\mathrm{Her}-2 / \mathrm{Neu}$ and the binding of trastuzumab leads to complement mediated killing of the HER-2 positive cells (Table 1) [11, 12]. Though there is evidence of a significant prolongation in disease-free survival in women receiving trastuzumab and chemotherapy compared to those receiving chemotherapy alone, it produces four types of toxicities which need special clinical attention [13].
(1) Cardiomyopathy. There is a 4- to 6-fold increase in the incidence of symptomatic left ventricular myocardial dysfunction (decline in left ventricular ejection fraction) among patients receiving Herceptin as a single agent or in combination therapy compared with those not receiving Herceptin. Moreover, it causes cardiac arrhythmias, hypertension, disabling cardiac failure, cardiomyopathy, and cardiac death.

(2) Infusion Reactions. Serious and fatal infusion reactions have been reported. Infusion reactions consist of a symptom complex characterized by fever and chills and on occasion included nausea, vomiting, pain (in some cases at tumor sites), headache, dizziness, dyspnea, hypotension, rash, and asthenia. Severe reactions including bronchospasm, anaphylaxis, angioedema, hypoxia, and severe hypotension were usually reported during or immediately following the initial infusion.

(3) Chemotherapy Induced Neutropenia. In randomized, controlled clinical trials the per-patient incidences of NCI CTC Grade 3 to 4 neutropenia and of febrile neutropenia were higher in patients receiving Herceptin in combination with myelosuppressive chemotherapy as compared to those who received chemotherapy alone.

(4) Pulmonary Toxicity. Herceptin use can result in serious and fatal pulmonary toxicity. Pulmonary toxicity includes dyspnea, interstitial pneumonitis, pulmonary infiltrates, pleural effusions, noncardiogenic pulmonary edema, pulmonary insufficiency and hypoxia, acute respiratory distress syndrome, and pulmonary fibrosis. Such events can occur as sequelae of infusion reactions [14].

Though both drugs are being in clinical use as antiangiogenic drugs, their pharmacological evaluation specially the efficacy and/or toxicity assessment in quantitative terms has not been evaluated. Previously, the molecular rationale of off-target toxicity of different drugs is established by using molecular docking interaction method between an array of receptors present within the physiological system and different adjuvant drugs of breast cancer [15]. Using the same 
approach, pharmacological assessment of different arsenic chelator drugs is evaluated [16]. The present work investigates the molecular portrayals in quantitative terms on the efficacy as well as the above-mentioned side effects caused by different antibody based antiangiogenic drugs.

\section{Materials and Methods}

2.1. Software and Database. For the present work we have used Hex, an open source, freely available software for academic use. The present work is done with the data resources that are available in the public domain. Table 2 provides the source of data availability used in this study. The Protein Data Bank (PDB) (http://www.rcsb.org/) is a worldwide repository for the processing and distribution of 3D biological macromolecular structure data [17]. Protein structures may be downloaded from the site with specific keywords or a PDB alphanumeric filename. The Drug Bank (http://redpoll.pharmacy.ualberta.ca/drugbank/) database [18] is a unique bioinformatics and cheminformatics resource that combines detailed drug (i.e., chemical, pharmacological, and pharmaceutical) data with comprehensive drug target (i.e., sequence, structure, and pathway) information. The database contains nearly 4300 drug entries including >1000 FDA-approved small molecule drugs, 113 FDA-approved biotech (protein/peptide) drugs, and $>3000$ experimental drugs. Additionally, more than 6000 protein (i.e., drug target) sequences are linked to these drug entries. Each Drug Card entry contains more than 80 data fields with half of the information being devoted to drug/chemical data and the other half devoted to drug target or protein data.

2.2. Molecular Docking. Molecular docking is performed using Hex program. Molecular docking helps in predicting the intermolecular interactions after forming an intermolecular complex between two constituent molecules. It uses spherical polar Fourier (SPF) correlations to accelerate the calculations. Using this program we have docked between receptor/enzyme and AAG drug (antibody)/drug in different combinations $[19,20]$. Briefly the steps are as follows:

(1) From the File menu the Receptor and Ligand files were opened.

(2) When two molecules are loaded the scene origin is taken as the midpoint between the two molecular centroids. Although both receptor and ligand move during docking, generally more motion is assigned to the ligand and keeping the receptor fixed though this can be changed by pressing the Select origin button.

(3) There are few Protein-Protein software programs that give output in terms of energy; Hex is an exception although the energy output is closer to internal energy $(\Delta U)$. The energy is a function of the distance range chosen by the two origins and also the angles between them. So $E=f(x, y, z)$ and $E=f(\varphi, \psi, \theta)$.

(4) Now the relation between $\Delta G$ and $\Delta U$ can be obtained from thermodynamics. We know entropy $S$, enthalpy $H$, and Gibb's free energy $\Delta G=\Delta H-T \Delta S$. So at constant pressure, $\Delta G=\Delta U+P \Delta V-T \Delta S$. Hex considers only $E_{\text {total }} \Delta U$ (total internal energy) and tends to neglect the entropy term that is the hydrophobic interactions; however, this corresponds as error at 100's of (+ or -) $\mathrm{KJ} / \mathrm{mol}$ compared to experimental condition [21].

(5) After the Docking is activated from the Controls menu, the Energy is output together with the diagram of the docked complex.

2.3. Visualization of Drug-Protein and Protein-Protein Complexes. For viewing the results of hex docking, we used Hex itself. After docking, the distance between two different molecules is also determined.

\section{Results}

Rigorous docking experiments had been performed to assess the comparative efficacy between two clinically used AAG drugs and their cross-reactivity to different receptors and/or enzymes within the physiological system. The AAG drugs, the receptors, and the corresponding docking results are listed in Table 3.

Our docking study reveals that the binding affinity of bevacizumab to VEGF receptor (-741.08) is much higher than its binding to Her-2/Neu receptor (-376.03). Binding affinity of trastuzumab to VEGF receptor $(-721.08)$ is higher than Her-2/Neu receptor (-562.51) though it is much higher than the binding affinity of bevacizumab to Her-2/Neu receptor (-376.03) (Table 3) (Figure 1).

Both AAG drugs bind to beta-2, tyrosine kinase (TK) receptor, and NO synthase which indicate their antiangiogenic potentiality. Interestingly, compared to bevacizumab, trastuzumab has the higher binding affinity to beta-1, beta2 , tyrosine kinase receptors, and NO synthase. This result may indicate that antiangiogenic potentiality of trastuzumab may be higher than bevacizumab. However, these results also indicate the reason of hypertension and cardiac myopathy in trastuzumab treated patients and why they need special attention when they show symptoms of hypertension and cardiac myopathy. Interestingly, the majority of cases, binding affinity of both the AAG drugs to different receptors/enzymes, are much higher compared to the known ligands (antagonist/agonist) to those receptors/enzymes. Trastuzumab has the highest binding towards NO synthase (-743.53) among the studied receptor/enzyme molecules in this work. Contrarily, bevacizumab has much less binding affinity towards NO synthase compared to their known ligands (antagonist/agonist); therefore there is much less chance of cardiac myopathy by this drug (Table 3) (Figure 2). Moreover, this data also indicates that, in bevacizumab treated patients, synergistic application of immunotherapy is also possible.

Bevacizumab does not have any binding affinity to angiotensin II type I (AT 1) and beta-1 receptor. However, binding affinity of bevacizumab is very close to angiotensin converting enzyme (ACE) antagonist lisinopril. Compared to trastuzumab it has also less affinity for ACE; so the cause 
TABLE 2: Sources of PDB files used in the study.

\begin{tabular}{|c|c|c|}
\hline Receptor/drug & Code/accession number & Website reference \\
\hline Androgen receptor & PDB ID 2YHD (edited) & http://www.rcsb.org/pdb/home/ \\
\hline Beta-1 receptor & PDB ID 2VT4 (edited) & $"$ \\
\hline Beta- 2 receptor & PDB ID 2R4R (edited) & $"$ \\
\hline $\begin{array}{l}\text { Dopamine-2 } \\
\text { (D2) receptor }\end{array}$ & PDB ID 2YOU (edited) & $”$ \\
\hline Estrogen- $\alpha($ ER- $\alpha)$ receptor & PDB ID 1X7E (edited) & $"$ \\
\hline GABA-A receptor & PDB ID 3D32 (edited) & $”$ \\
\hline GABA-B receptor & PDB ID 1SRZ (edited) & $”$ \\
\hline Histamine $(\mathrm{H} 2)$ receptor & Univ. of Michigan & http://www.personal.umich.edu/ him/H2A.htm ${ }^{*}$ \\
\hline $\begin{array}{l}\text { Angiotensin II } \\
\text { type } 1 \text { (AT 1) receptor }\end{array}$ & PDB ID 3D0G (edited) & http://www.rcsb.org/pdb/home/ \\
\hline $\begin{array}{l}\text { Nitric oxide synthase } \\
\text { (NOS) }\end{array}$ & PDB ID 1ED5 (edited) & $”$ \\
\hline Tyrosine kinase (TK) & PDB ID 1M17 (edited TK domain of EGF) & http://www.rcsb.org/pdb/home/ \\
\hline $\begin{array}{l}\text { Adrenoceptor alpha } 2 \mathrm{a} \\
\text { (ADRA2A) }\end{array}$ & PDB ID 1HLL & $”$ \\
\hline $\begin{array}{l}\text { Angiotensin converting } \\
\text { enzyme (ACE) }\end{array}$ & PDB ID 4UFA & $”$ \\
\hline Ca-channel & PDB ID 1T3L & $”$ \\
\hline $\mathrm{Her}-2 / \mathrm{Neu}$ & PDB ID 1 S78 (edited) & http://www.rcsb.org/pdb/home/ \\
\hline $\begin{array}{l}\text { VEGF } \\
\text { (vascular endothelial } \\
\text { growth factor)-C }\end{array}$ & PDB ID 2XIX (edited) & $”$ \\
\hline Angiotensin II & PDB ID 1ZV0 (edited) & $”$ \\
\hline $\begin{array}{l}\text { NNA } \\
\text { (N-nitro-L-arginine) }\end{array}$ & PDB ID 8NSE & ” \\
\hline Imatinib & Primary Acc. number DB00619 & http://www.drugbank.ca \\
\hline Cyproterone & Primary Acc. number DB04839 & $"$ \\
\hline Nandrolone & Primary Acc. number DB00984 & $"$ \\
\hline Propranolol & Primary Acc. number DB00571 & $”$ \\
\hline Epinephrine & Primary Acc. number DB00668 & $”$ \\
\hline Risperidone & Primary Acc. number DB00734 & $"$ \\
\hline Cabergoline & Primary Acc. number DB00248 & $”$ \\
\hline Flumazenil & Primary Acc. number DB01205 & $”$ \\
\hline Diazepam & Primary Acc. number DB00829 & $"$ \\
\hline Tamoxifen & Primary Acc. number DB00675 & http://www.drugbank.ca \\
\hline Ethinyl estradiol & Primary Acc. number DB00977 & $"$ \\
\hline Saclofen & $\begin{array}{c}\text { Marvin sketched at } \\
\text { http://www.chemaxon.com and saved as } \\
\text { pdb }\end{array}$ & http://www.chemaxon.com \\
\hline Baclofen & Primary Acc. number DB00181 & http://www.drugbank.ca \\
\hline Ranitidine & Primary Acc. number DB00863 & $”$ \\
\hline Betazole & Primary Acc. number DB00272 & $”$ \\
\hline Losartan & Primary Acc. number DB00678 & $”$ \\
\hline Yohimbine & Primary Acc. number DB01392 & $"$ \\
\hline Clonidine & Primary Acc. number DB00575 & $"$ \\
\hline Lisinopril & Primary Acc. number DB00722 & $"$ \\
\hline Nifedipine & Primary Acc. number DB01115 & $"$ \\
\hline Bevacizumab/Avastin & Primary Acc. number DB00112 & $”$ \\
\hline Trastuzumab/Herceptin & Primary Acc. number DB00072 & $”$ \\
\hline Substance P & Primary Acc. number DB05875 & $”$ \\
\hline
\end{tabular}

* accessed on 4-Dec-2011. 


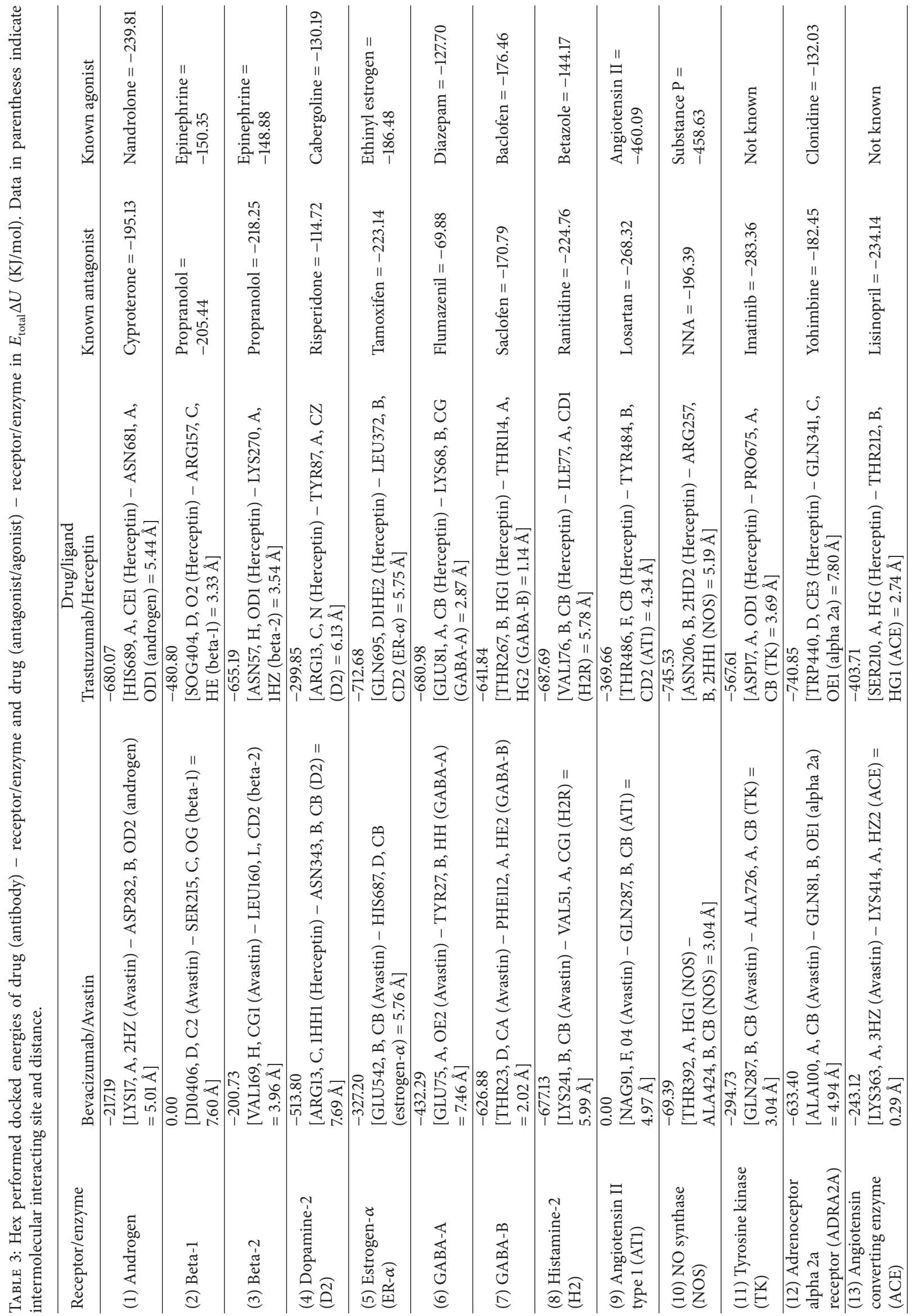




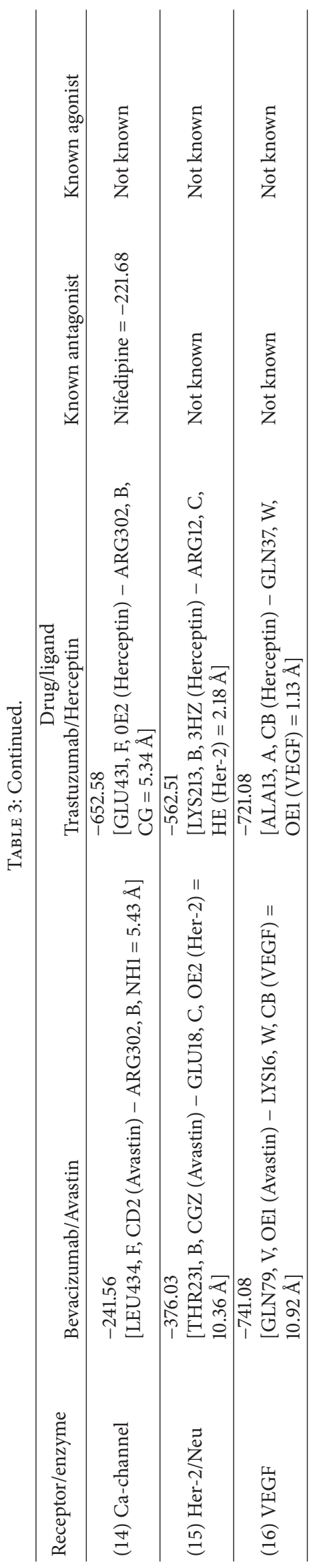




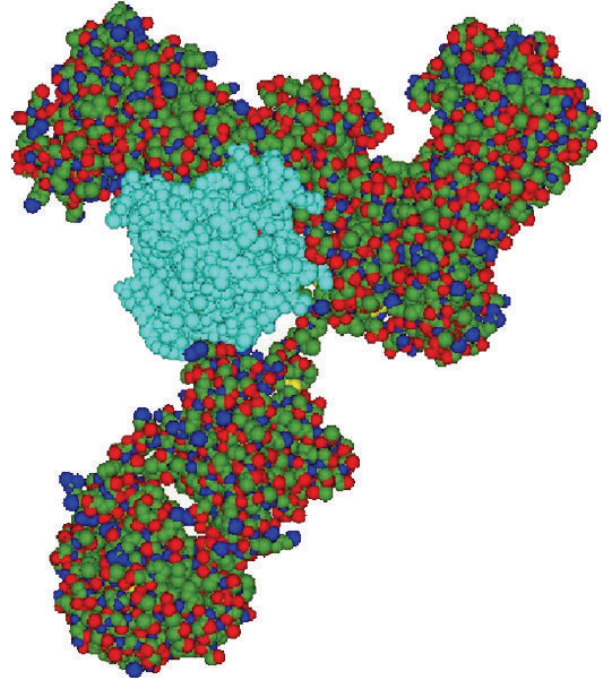

(a)

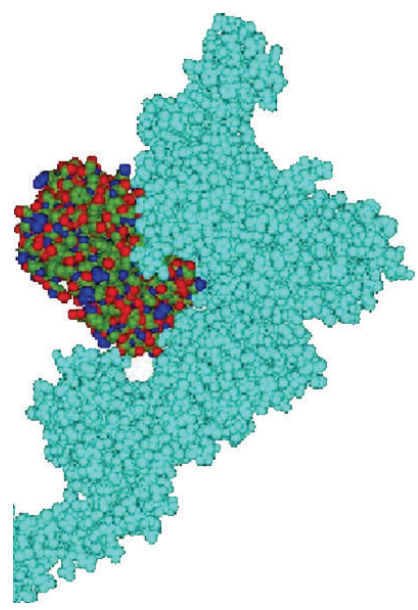

(c)

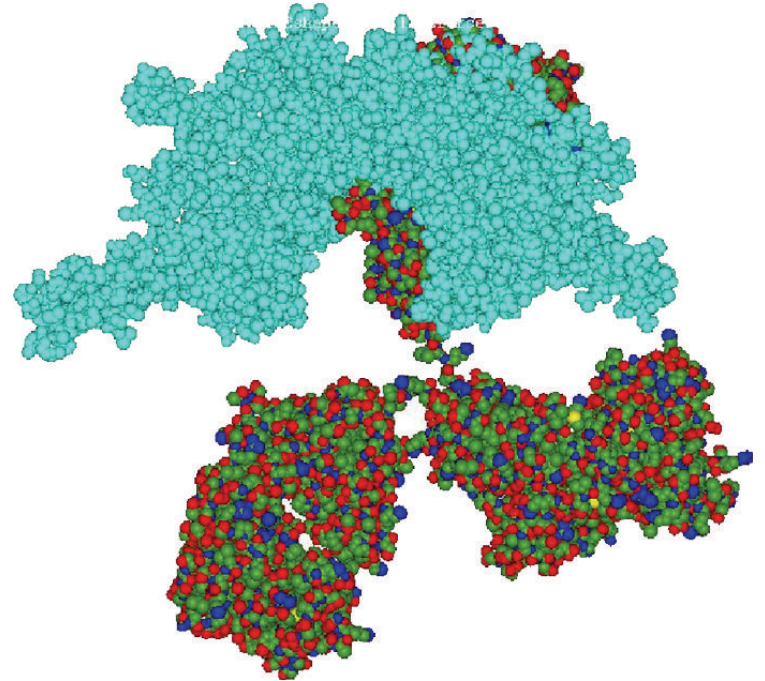

(b)

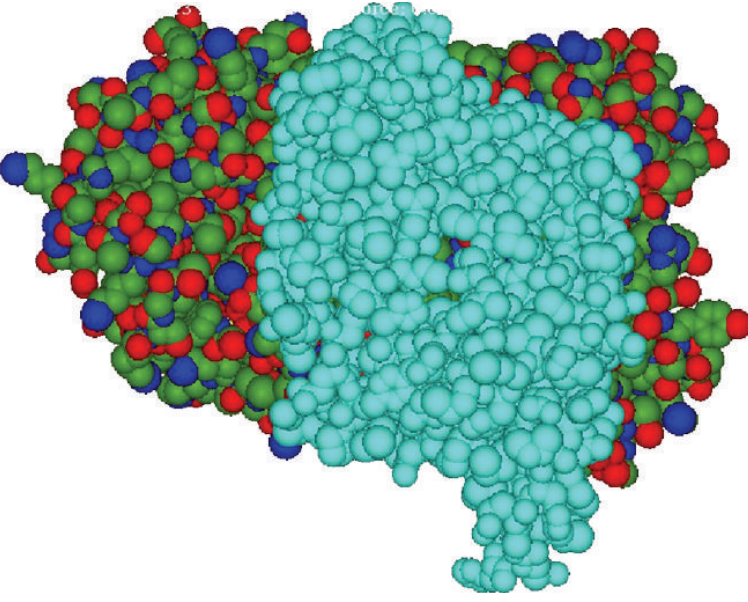

(d)

Figure 1: Docked conformation of bevacizumab to VEGF (a) and Her-2/Neu receptor (b), and trastuzumab to Her-2/Neu (c) and VEGF receptor $(\mathrm{d})$; the receptor protein is in cyan color.

of observed hypertension by the use of bevacizumab may be due to destruction of microvessels or it may act as an agonist for ACE, whereas higher binding of trastuzumab to ACE may indicate the cause of infusion reaction by trastuzumab [5].

Both AAG drugs showed much higher binding affinity towards estrogen receptor (ER); hence, both of them would be effective in estrogen positive breast/ovarian cancer. Compared to bevacizumab, trastuzumab has increased binding affinity to ER-alpha; therefore trastuzumab may also be more effective in controlling of estrogen positive breast/ovarian cancer (Table 3) (Figure 3). The docked conformation of two AAG drugs to the androgen receptor (AR) showed that the binding affinity of two AAG drugs has much higher binding affinity to it compared to its known ligands. These results indicate that AAG drugs are effective in other cancers related to steroidal hormones. For this type of receptor trastuzumab also showed higher binding affinity than bevacizumab.

Our docking results also reveal that both AAG drugs have a higher binding affinity towards dopamine-2 receptor [bevacizumab $(-513.8)>$ trastuzumab $(-299.85)$ ]; this data indicates why the symptom of nausea is more pronounced with bevacizumab treatment than with trastuzumab (Figure 4). Both the AAG drugs have almost equal binding affinity to histamin-2 receptor and both have much higher binding affinity with respect to known ligands (antagonist and agonist). Similar trend is also seen for both types of GABA receptors with lesser for bevaczumab to GABA-A (Table 3). Simulation data also suggest that both AAG drugs 


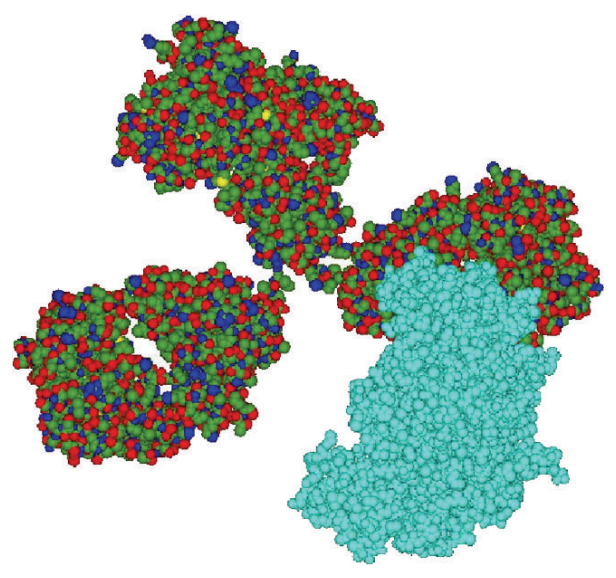

(a)

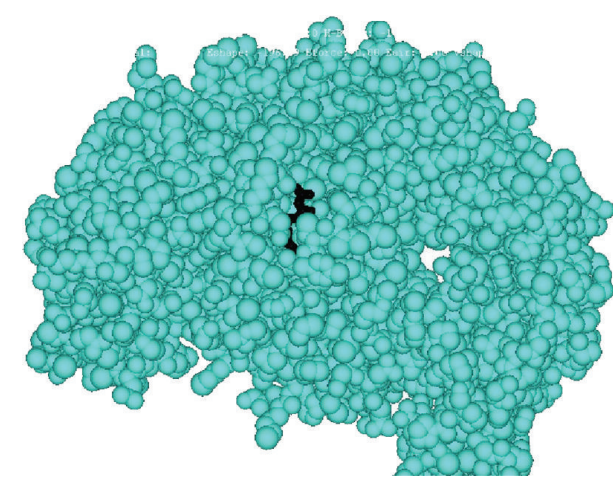

(c)

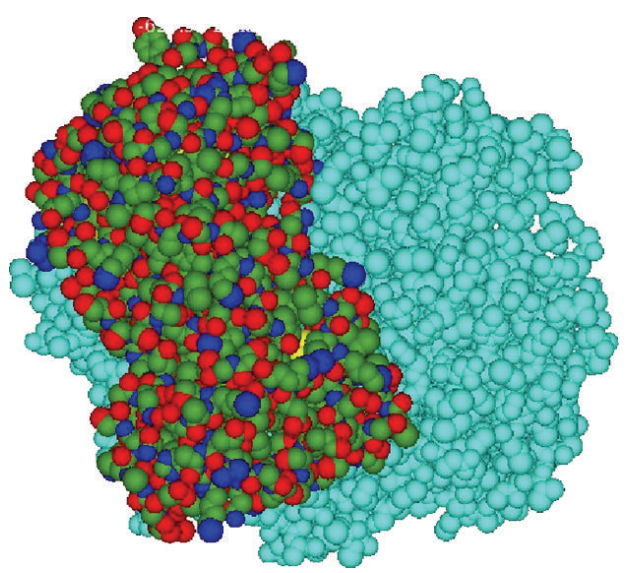

(b)

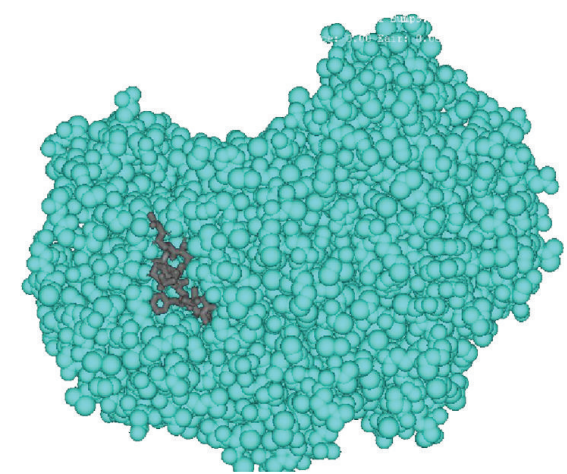

(d)

FIGURE 2: Docked conformation of bevacizumab (a), trastuzumab (b), NNA (c), and substance P (d) to NO synthase (in cyan color).

have very high binding (compared to its known ligands) towards adrenoceptor alpha 2 a receptor (ADRA2A), which is very important receptor for retinal functioning. So this study also reveals the molecular rationale of ocular dysfunction by AAG drugs.

\section{Discussion}

Long-term treatment is necessary for different cancers. But conventional chemotherapies have toxic side effect. Hence there is a search for specificity in cancer treatment. It is expected that monoclonal antibody based drugs may provide that way out. Though both bevacizumab and trastuzumab are FDA-approved antiangiogenic drugs in clinical use for the therapy of a wide variety of cancers, their efficacy and/or selection for clinical use are in empirical state. There are scanty report regarding their selection and drug scheduling for different cancers. Different clinical trials suggest for combination therapy even with the conventional chemotherapy. So our computational prediction is useful due to scanty data availability.
Though different analytical and simulation based studies showed that the efficacies of such drugs may have better in control of tumor growth, different clinical reports suggest that these monoclonal antibody based drugs do not qualify towards the expected criteria of overcoming the physiological toxicity $[22,23]$. This, in turn, may limit the wider acceptance of AAG drugs for cancer treatment. The issue of drug related toxicity has become the focal theme with the rise of systems biology and for a decade long this issue has been addressed in the area of cancer systems biology which reinforces the need of theoretical as well as simulation study [24-30].

Previously different docking studies (using Autodock and/or Hex) have proved the efficacy of macromolecular interactions as well as drug-macromolecular interactions, where docking results indicate the fact that the more the negative energy, the more the binding efficacy $[15,16,19-$ 21]. Previous work also suggests that off-target toxicity can be studied through docking based method and, for this, docked result between a drug and its off-target is compared with the docked result between the same target and its known ligand/drug. If the two results are similar or near to similar 


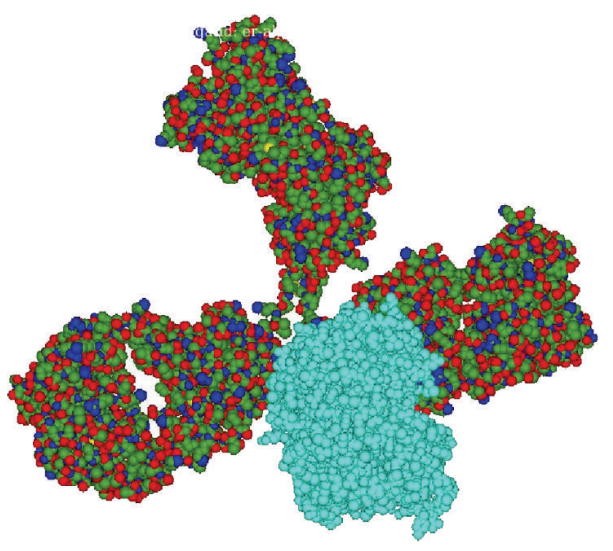

(a)

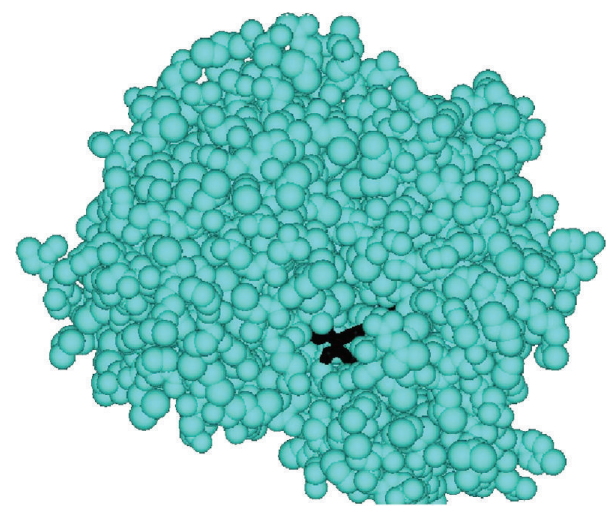

(c)

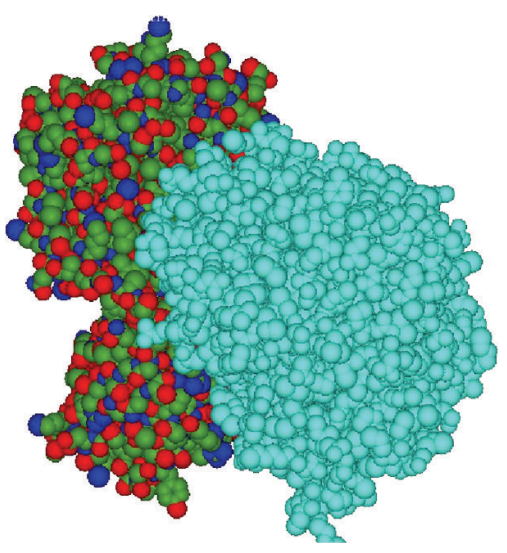

(b)

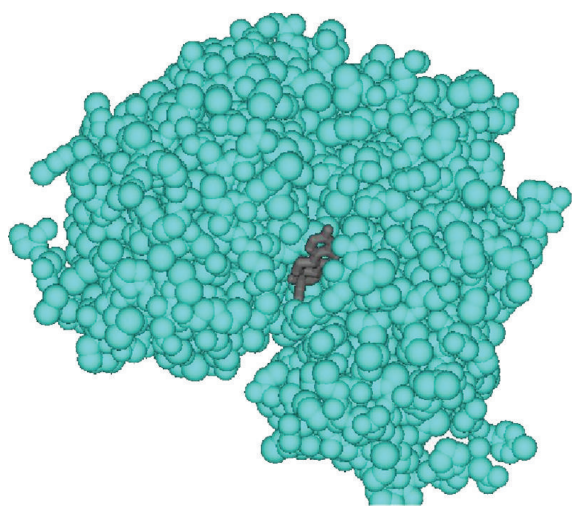

(d)

Figure 3: Docked conformation of bevacizumab (a), trastuzumab (b), tamoxifen (c), and ethinyl estradiol (d) to estrogen receptor alpha (in cyan color).

then off-target binding can be concluded. If former result is greater than or near the value of the latter then off-target toxicity is possible. Again comparison of binding data of drug with off-target, target with known antagonist/agonist, and the clinical findings/reports may hint towards the molecular rationale of pharmacological/toxicological mechanisms [15].

The present docking based study indicates that trastuzumab has better antiangiogenic potentiality compared to bevacizumab; however, it has much more side effects on the cardiovascular system. The major problems of using AAG drugs are nausea, hypertension, gastric ulceration, and ocular damage. Our docking study reveals its molecular rationale as the binding affinity of both AAG drugs on D2, beta-2, GABA, ACE, Ca-channel, H2, and ADRA2A; most importantly trastuzumab has the highest binding affinity for NOS; this may be the probable cause that in trastuzumab treatment cardiomyopathy needs special attention. Our data also indicate that, in bevacizumab treated patients, synergistic application of other immunotherapy is also possible.

This in silico study may provide the molecular rationale of toxicity within the physiological system and hints towards the probable cause of pathophysiological alteration with the application of AAG drugs in patients. With the availability of time dependent data related to the onset toxicological symptoms by AAG drugs, a correlation study between the two would then be beneficial in the future particularly for the calibration of drug scheduling. Hence, generation of dynamical database regarding cancer treatment along with the onset of toxicological symptoms in humans (for different drugs) is needed. Towards this aspect this study has the significance.

\section{Conflict of Interests}

The authors declare that there is no conflict of interests. 


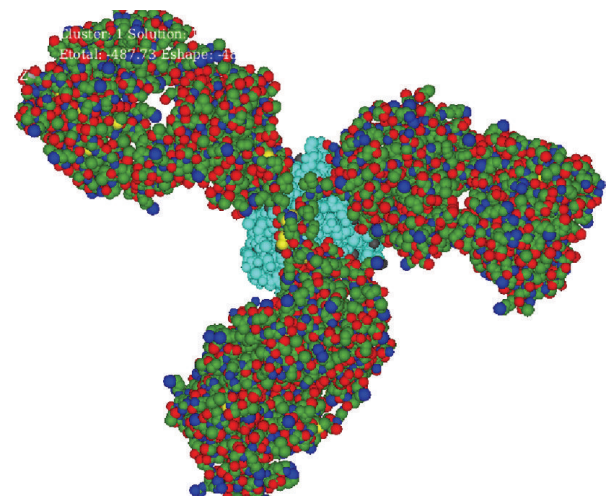

(a)

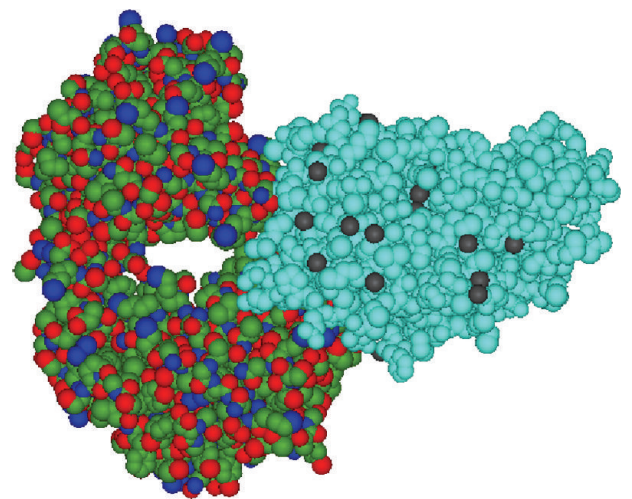

(b)

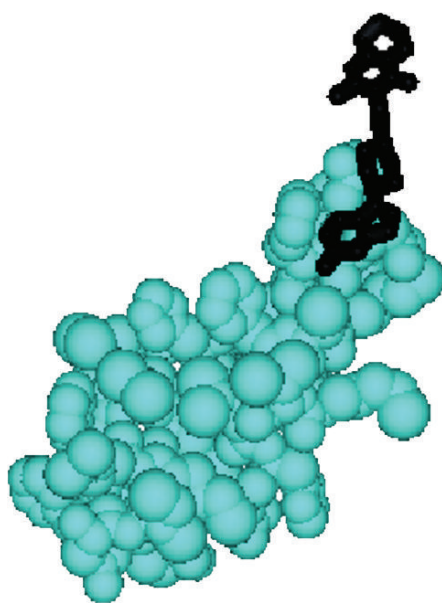

(c)

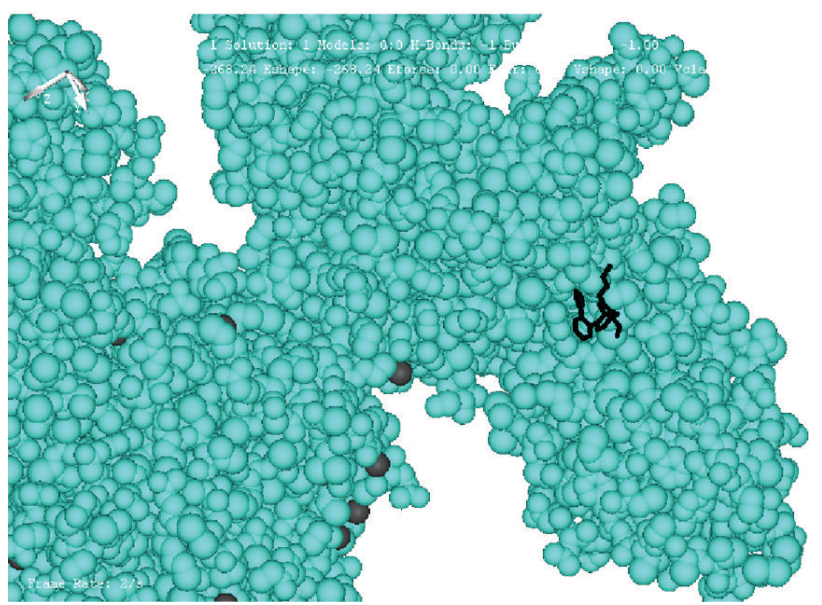

(d)

Figure 4: Docked conformation of bevacizumab (a), trastuzumab (b), risperidone (c), and cabergoline (d) to dopamine-2 receptor (in cyan color).

\section{Acknowledgment}

The authors acknowledge the critical comments of the eminent founder members of Society for Systems Biology \& Translational Research, India.

\section{References}

[1] D. H. Ausprunk and J. Folkman, "Migration and proliferation of endothelial cells in preformed and newly formed blood vessels during tumor angiogenesis," Microvascular Research, vol. 14, no. 1, pp. 53-65, 1977.

[2] W. Risau, "Mechanisms of angiogenesis," Nature, vol. 386, no. 6626, pp. 671-674, 1997.

[3] C. Schettino, M. A. Bareschino, V. Ricci, and F. Ciardiello, "Erlotinib: an EGF receptor tyrosine kinase inhibitor in nonsmall-cell lung cancer treatment," Expert Review of Respiratory Medicine, vol. 2, no. 2, pp. 167-178, 2008.

[4] J.-M. Lee, G. A. Sarosy, C. M. Annunziata et al., "Combination therapy: intermittent sorafenib with bevacizumab yields activity and decreased toxicity," British Journal of Cancer, vol. 102, no. 3, pp. 495-499, 2010.

[5] S. Banerjee, M. Dowsett, A. Ashworth, and L.-A. Martin, "Mechanisms of Disease: angiogenesis and the management of breast cancer," Nature Clinical Practice Oncology, vol. 4, no. 9, pp. 536-550, 2007.

[6] E. A. Donovan and S. Kummar, "Targeting VEGF in cancer therapy," Current Problems in Cancer, vol. 30, no. 1, pp. 7-32, 2006.

[7] F. Gurevich and M. A. Perazella, "Renal effects of antiangiogenesis therapy: update for the internist," The American Journal of Medicine, vol. 122, no. 4, pp. 322-328, 2009.

[8] S. Hapani, D. Chu, and S. Wu, "Risk of gastrointestinal perforation in patients with cancer treated with bevacizumab: a metaanalysis," The Lancet Oncology, vol. 10, no. 6, pp. 559-568, 2009.

[9] S. S. Shord, L. R. Bressler, L. A. Tierney, S. Cuellar, and A. George, "Understanding and managing the possible adverse effects associated with bevacizumab," American Journal of Health-System Pharmacy, vol. 66, no. 11, pp. 999-1013, 2009. 
[10] D. Ribatti, V. Longo, and A. Vacca, "Antiangiogenic treatment of hematological tumors," in Anti-Angiogenesis Drug Discovery and Development, A. Rahman and M. I. Choudhary, Eds., vol. 1, pp. 1-11, Bentham Science, Sharjah, UAE, 2011.

[11] CenterWatch Drug Information Herceptin, 2010, http://www .centerwatch.com/drug-information/fda-approved-drugs/ year/2010.

[12] Funakoshi, Japan, 2015, http://www.funakoshi.co.jp/data/datasheet/ ABD/HCA176.pdf.

[13] National Cancer Institute, FDA Approval for Trastuzumab, 2015, http://www.cancer.gov/about-cancer/treatment/drugs/fdatrastuzumab.

[14] Genentech, "FDA full precribing information, warnings and precautions," 2010, http://www.accessdata.fda.gov/drugsatfda _docs/label/2010/103792s5250lbl.pdf.

[15] S. Mukherjee and D. Majumder, "Computational molecular docking assessment of hormone receptor adjuvant drugs: breast cancer as an example," Pathophysiology, vol. 16, no. 1, pp. 19-29, 2009.

[16] D. Majumder and S. Mukherjee, "Molecular docking assessment of efficacy of different clinically used arsenic chelator drugs," Journal of Computational Medicine, vol. 2013, Article ID 396768, 8 pages, 2013.

[17] H. M. Berman, J. Westbrook, Z. Feng et al., "The protein data bank," Nucleic Acids Research, vol. 28, no. 1, pp. 235-242, 2000.

[18] D. S. Wishart, C. Knox, A. C. Guo et al., "DrugBank: a comprehensive resource for in silico drug discovery and exploration," Nucleic Acids Research, vol. 34, supplement 1, pp. D668-D672, 2006.

[19] B. Das and D. Majumder, "Interactions among MARM binding factors," in Proceedings of the World Congress on Information and Communication Technologies (WICT '12), A. Abraham, S. M. Thampi, S. Pal et al., Eds., pp. 191-196, Trivandrum, India, October-November 2012.

[20] B. Das and D. Majumder, "Interactions of transcription factors in HLA class I transcriptosome," International Journal of Computer Information Systems, vol. 6, pp. 592-602, 2014.

[21] D. Ritchie, "Hex 8.0.0 user manual," 1996, http://hex.loria.fr/ manual800/hex_manual.pdf.

[22] A. Mukherjee and D. Majumder, "Dynamical model for assessment of anti-angiogenic therapy of cancer," Molecular BioSystems, vol. 6, no. 6, pp. 1047-1055, 2010.

[23] P. K. Dhar and D. Majumder, "A composite synergistic systems model for exploring the efficacies of different chemotherapeutic strategies in cancer," Computational Biology Journal, vol. 2013, Article ID 301369, 26 pages, 2013.

[24] D. Majumder and A. Mukherjee, "Patho-physiologically based logistics for treatment of cancer," Journal of Biological Systems, vol. 14, no. 4, pp. 631-650, 2006.

[25] D. Majumder and A. Mukherjee, "Multi-scale modeling approaches in systems biology towards the assessment of cancer treatment dynamics: adoption of middle-out rationalist approach," Advances in Cancer Research \& Treatment, vol. 2013, Article ID 587889, 26 pages, 2013.

[26] D. Majumder and A. Mukherjee, "Mathematical modelling of toxicity-related trade-offs in metronomic chemotherapy," IET Systems Biology, vol. 1, no. 5, pp. 298-305, 2007.

[27] O. Wolkenhauer, "Defining systems biology: an engineering perspective," IET Systems Biology, vol. 1, no. 4, pp. 204-206, 2007.
[28] A. Mukherjee and D. Majumder, "Mathematical modelling for the assessment of the effect of drug application delays in metronomic chemotherapy of cancer due to physiological constraints," BioSystems, vol. 91, no. 1, pp. 108-116, 2008.

[29] D. Majumder and A. Mukherjee, "A passage through systems biology to systems medicine: adoption of middle-out rational approaches towards the understanding of clinical outcome in cancer therapy," Analyst, vol. 136, no. 4, pp. 663-678, 2011.

[30] D. Majumder, "Assessment for possible drug application delays in MCT strategy due to pathophysiological constraints of cancer," in Proceedings of the IEEE World Congress on Information and Communication Technologies (WICT '12), A. Abraham, S. M. Thampi, S. Pal et al., Eds., Paper 233, IEEE Catalog Number: CFP1268R-CDR, pp. 4673-4804, Trivandrum, India, OctoberNovember 2012. 

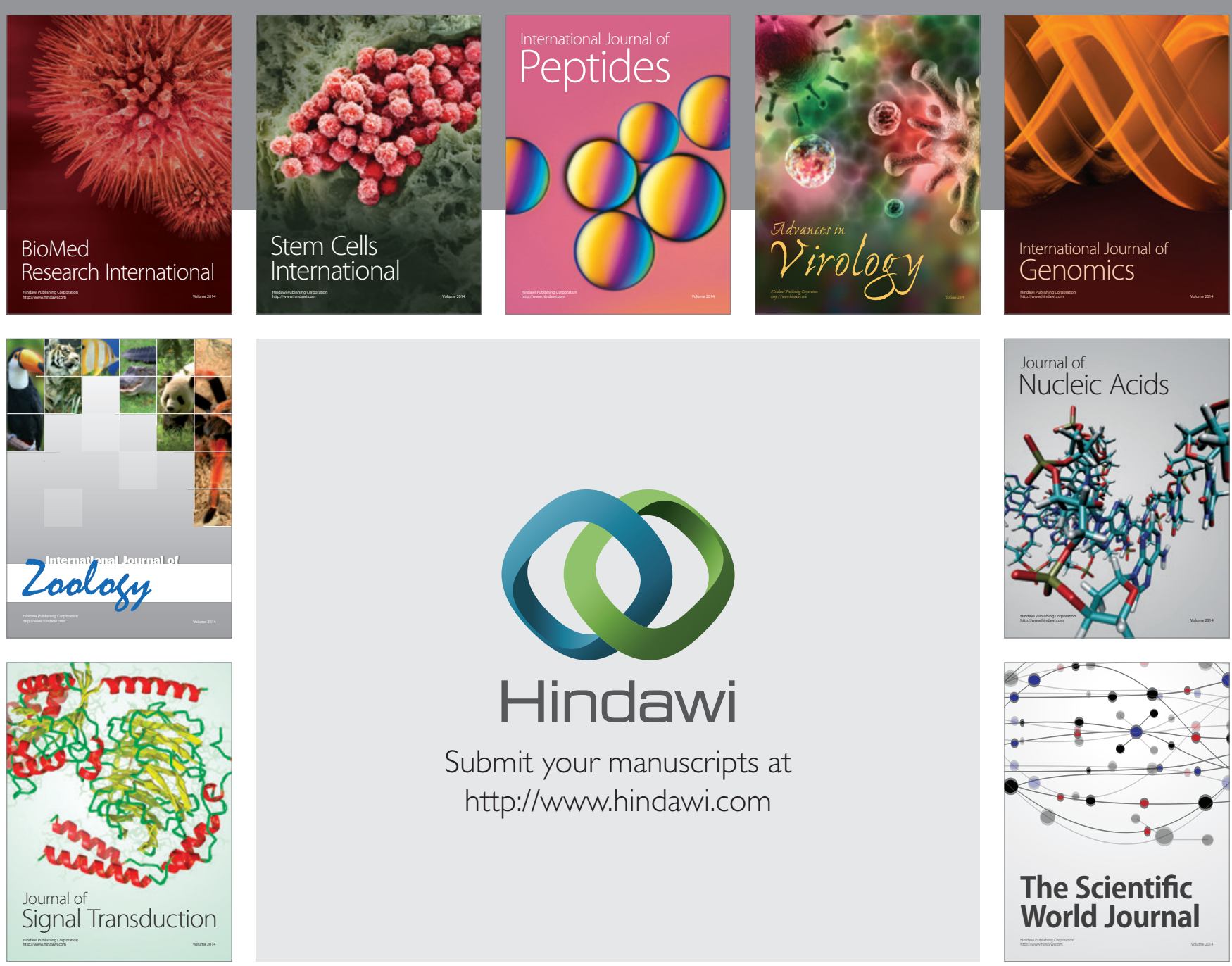

Submit your manuscripts at

http://www.hindawi.com
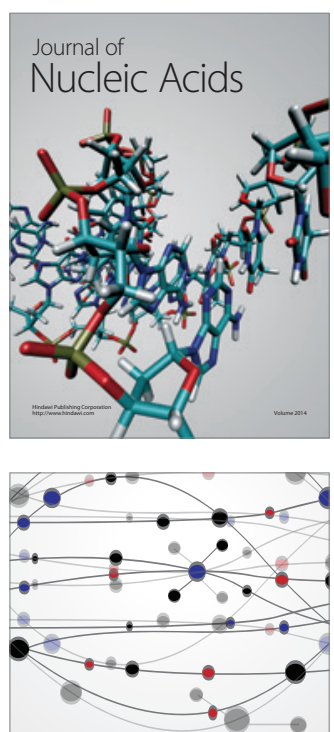

The Scientific World Journal
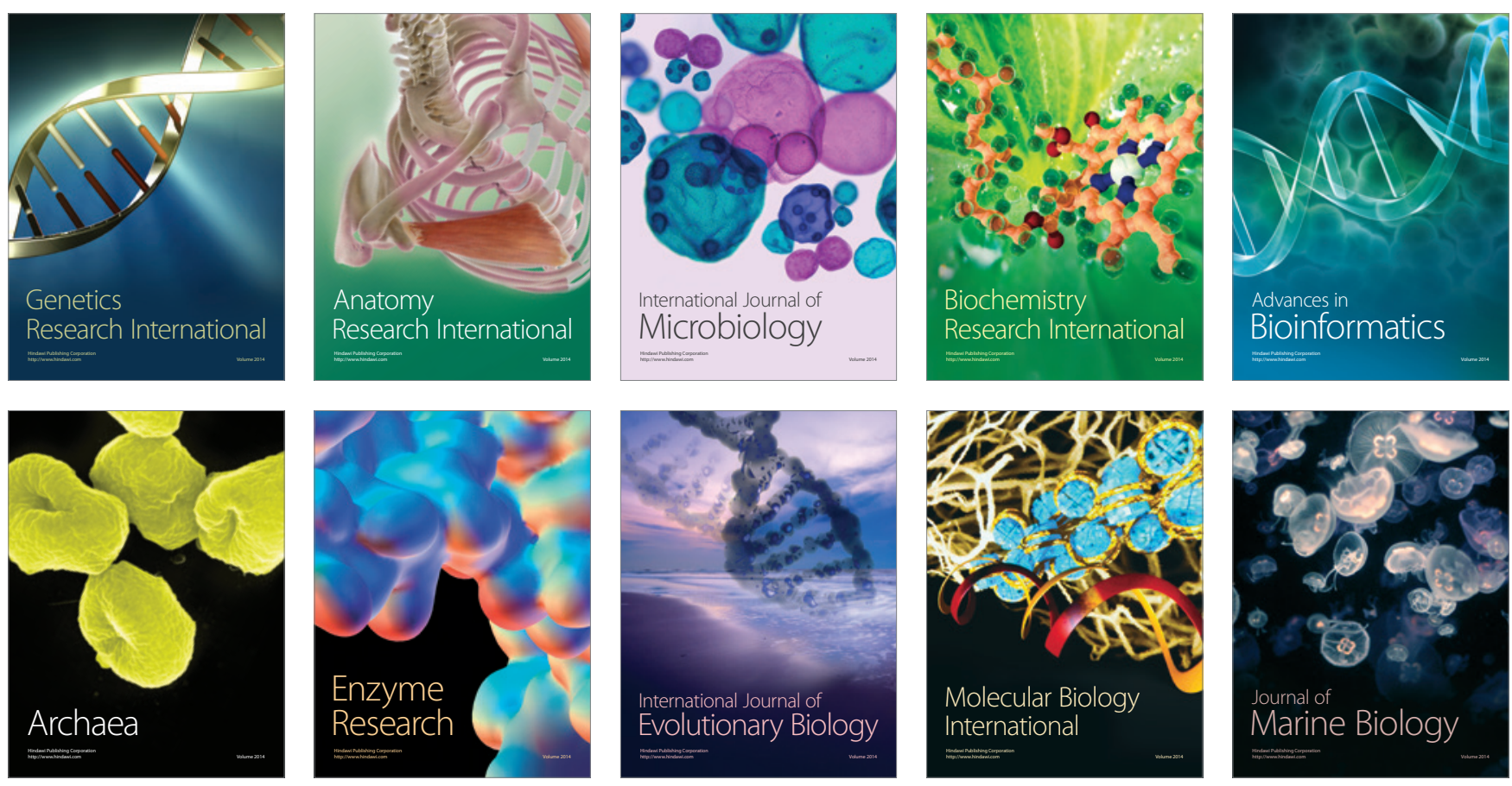